Dr. J. Rilus Eastman ${ }^{4}$ of Indianapolis, reports that his results have been much better since he has been removing the appendix in every case of this character; he most heartily commends the method.

In brief, the technic of the procedure recommended is the following: The incision, regardless of the location of the abscess, is made through the right rectus muscle, and the peritoneal cavity is freely opened. A small gauze pack is tucked into the upper angle of the wound and so adjusted as to prevent any extravasation of pus toward the median line. A gauze pack is adjusted also from the upper angle of the wound to the lower pelvis to prevent any extravasation toward the left. The abscess cavity is then freely opened with the fingers unless opened by the peritoneal incision, and the pus is rapidly mopped up with gauze sponges. The appendix is then exposed and removed. All adhesions separating the abscess cavity from the lower pelvis are now freely separated and the pus or seropus which is usually found in the lower peritoneal pouch is rapidly removed by sponges. A large split rubber tube, earrying a loose-fitting wick of iodoform gauze, is inserted through the lower angle of the wound to the bottom of the rectovesical pouch, care being taken that it does not, at the lower end, impinge on an intestinal coil. The protecting gauze is removed and the wound closed down to the tube with interrupted through-and-through sutures of silkworm-gut. In these cases a continuouslayer suture is greatly inferior to a through-andthrough suture because of the danger of infection of the wound. As drainage of the peritoneal cavity can be maintained only for a relatively short time, this drainage must, in cases of this character, be very free, and the use of a large tube, placed as above described, secures this result.

The patient is then placed in bed, the head of which is elevated at least 30 inches from the floor. The patient is turned on his right side inclining toward the prone position, and a special nurse, instructed to see that the right-side posture is maintained for at least twenty-four hours, should be placed in charge. Proctoclysis is immediately begun and continued as long as needed in each case. Water is allowed at once, but in small quantities. Morphin is given whenever necessary to control pain. No nourishment is given for at least forty-eight hours and then it is given sparingly.

The cases which furnish the material for the conclusions reached in this article represent an unbroken series of similar treatments, as I have removed the appendix in every case of appendicitis with localized abscess, since the first one of the series was removed.

Many of these patients were operated on in their homes, frequently under most unfavorable conditions, and I feel that the series furnishes a severe test of the method.

In considering the mortality of this procedure, which as previously remarked, has been, in this series, 1.2 per cent., we not only must compare with the primary mortality of similar cases treated by drainage alone, but also must add to this latter mortality that attending those cases in which because of recurrence of disease, a second or a third operation becomes necessarv. In my experience, not only has the mortality been greatly reduced by this plan of treatment, but also convalescence has been much shortened.

4. Eastman, J. Rilus: Impressions of Appendicitls and Sig. 4. Eastman, J. Rilus : Impressions of Appendicitis and
moidal Diverticulitis, Indianapolis Med. Jour., 1912, xv, 49 .
The procedure is not advocated for the operator with little experience in abdominal surgery; but it may be added, no man of little experience should attempt to operate on any acute suppurative process within the abdomen. The technic was, I freely admit, adopted as a routine measure with some misgiving at first, and I rather expected to be forced to discontinue it, at least in certain instances, but as experience with it increased and it was noted that adhesions in these cases could be freely separated, not only with impunity, but also under proper precautions, even with benefit, my faith in it has been strengthened and I no longer hesitate to offer it as a routine procedure to the competent surgeon, for I firmly believe that by its employment convalescence will be shortened, postoperative sequelae will occur less frequently and mortality be reduced.

\section{A CLINICAL STUDY OF ASTHMA}

\author{
B. C. DAVIES, M.D. \\ MONROVIA, CAL.
}

The purpose of this article is not to discuss asthma as a disease, but rather to treat it as a symptom, and to recite the results obtained in a series of cases based on this assumption.

Before proceeding to the case histories I would call attention to a review of the condition commonly called asthma and the classical theories generally accepted as to its cause.

Asthma is often designated as gastric, renal, nasal, bronchial, cardiac, etc. Two general classifications bring it down to cardiac and bronchial, though Osler says that, as a matter of fact, true asthma is rarely found in diseases of the heart; that while a dyspnea is present, it is not an asthmatic paroxysm, because it is an inspiratory instead of an expiratory interference.

Of special pathology there is practically none, such changes as are found being secondary, in the form of emphysema, bronchitis or dilatation of the right heart. The occurrence of asthma is paroxysmal and painless, stamping it at once a neurosis. It is characterized by spasm of the bronchi and the respiratory muscles, including also the diaphragm, and is followed by a vasomotor disturbance in the mucous membrane of the bronchi, large and small.

Briefly, the theories as to the cause of asthma, are as follows:

1. Spasm of the circular muscular fibers of the bronchial wall.

2. Hyperemia of the mucous membrane of the bronchioles.

3. Spasm of the diaphragm.

The first two are the most tenable, and, indeed, the results of investigations are causing, and justly, the first to be classified as the cause, with the second and third secondary in effect to the first, that is, resulting from the spasm of the bronchioles.

After disposing of the second and third theory, we then ask what produces the condition named in the first theory? The nearest answer yet offered is that it is a reflex from an irritation produced elsewhere in the body. As proof of this theory I wish to offer the following case histories compiled during the past two or three years, in the hope that more attention may be given to thorough physical examination of asthmatic sufferers. The cases cited are selected from different series of causes and are illustrative of what may cause an asthmatic paroxysm: 


\section{REPORT OF CASES}

UASE 1.-Mrs. F., an American, aged 32, has had the ordinary diseases of childhood. When 8 years of age she fell from a picket fence, alighting in a sitting posture. She was confined to bed for several days. The first menstruation, which was very painful, was at the age of 14 . At this time she experienced the first asthmatic attack. Attacks of asthma came with every subsequent menstruation, each menstruation being painful. At the age of 26 she married and har no asthma for the subsequent six years, during which time she bore three children. 'The youngest child is now nearly 3 , and for the past year the asthma has been increasing with each menstruation.

Examination.-Asthma here pointed so directly to a uterine disorder that a vaginal examination was made, and a condition found of retroversion with adhesions, probably due to the fall. The diagnosis of asthma reflex from uterine disorder was made.

Treatment.-A ventral fixation and ovariotomy were done April, 1912. Since the operation the patient has gained 15 to 20 pounds, with not a trace of asthma to date-May, 1913. The menstrual flow has been normal ever since. There have been four similar cases in this series, with three full recoveries. One showed no results.

Case 2 is of course rare but demonstrates the possibilities, and it is not by any means isolated in occurrence.

CASE 2,-Mr. G., aged 30 , is married. The history is negative except that the right testicle had not descended. At the age of 13 asthma developed and increased in severity until the patient was 18 years of age, when the attacks became terrific. The patient became a masturbator and reports that a severe attack followed each indulgence. At the time of the attack the patient complained of severe pain in the right inguinal region, which pain subsided in forty-eight to seventy-two hours and the asthma ceased shortly after. He was operated on for appendicitis five years ago. It was recommended that he have an exploratory abdominal section for investigation concerning the testicle which had not descended. The patient refused to act on the recommendation and returned to his home in the East. Two months Jater he wrote that his family physician had taken him to a surgeon because of excessive pain in the right inguinal region. An encysted testicle was removed. I have since heard from him twice and he is perfectly free from asthma. The operation was performed two years ago.

Case 3 illustrates a class that is probably the most common; at the same time it is the most easily diagnosed and responds readily to treatment.

CASE 3.-Mr. W., aged 65, has had asthma for fifteen years. His occupation is that of a broker. He is sedentary in his liabits and has been a heavy eater and drinker for many yedrs. There has been a great deal of constipation during the past twenty years; also indigestion with formation of gas and consequent pain. The amount of food was lessened. Drinking has been eliminated for the past five years. The patient is relieved of attacks of asthma when fasting or resting in bed for a few days.

Examination.-The blood-pressure was 180 . The urine was heavily loaded with indican and there was some albumin. Coarse râles were heard in the lungs and emphysema was present. The heart was irregular in action with a pulse-rate of 84. The abdomen was very protuberant. There was gastroptosis, descending 2 inches below the umbilicus. Enteroptosis was also marked. The abdomen was excessively tympanitic. Inguinal hernia has existed since the age of $\mathbf{3 5}$. A diagnosis of reflex asthma from abdominal ptosis and intes. tinal putrefaction was made.

Treatment.-An abdominal support was fitted very snugly supporting the walls firmly. The diet was regulated, proteids and coffee being eliminated almost entirely. Intestinal antiseptics were given and bowel movements were aided. Tincture of iodin in milk for high blood-pressure with rest in bed was preseribed. The result, ten days later, was a much lessened amount of indican, while the blood-pressure was 150 . The bowels moved daily and the patient said that breathing had not been so well or easily accomplished in ten years. The asthma had practically disappeared. Dyspnea had been almost continuous. One year las since elapsed (June, 1913), and the patient is still free from asthma unless dietetic errors occur, when asthma again presents itself.

Of this last class there have been fourteen cases. All have responded to treatment but two. One patient neglected to live up to the requirements and was eliminated from consideration. The other case has not been cleared up as yet, and probably will not be on this classification. Seven patients belonging to this class of cases have recovered entirely from their asthma. The remaining five are all much relieved. In all cases a return to the old methods of living, and the leaving off of abdominal support, precipitates an asthmatic attack. The intestinal antisepsis is no doubt the chief factor in the relief afforded; the belt, while essential, plays a secondary part.

As this article is being written a colleague reports an acute case.

CAse 4.- A man aged 45, awakened at 2 a. m. with a first attack of asthma. A canned-fish supper had been partaken of on the preceding evening. The attack was terminated not by morphin but by gastric lavage and a high enema.

The next case illustrates a large class and requires careful examination.

Case 5.-Miss S., aged 19, has had asthma since 7 years of age, following an attack of bronchitis. The paroxysms are almost continuous.

Examination.-The patient has adenoids and hypertrophied tonsils, also septum deviation. She is very anemic. The hemoglobin is 60 . There is chronic constipation. The urine is highly colored and heavily loaded with indican.

Treatment.-The adenoids and tonsils were removed. The septum deviation was corrected and the bowels were regulated through the diet. There was marked improvement in the asthmatic condition. The patient is subject yet to mild and brief attacks, though she is gaining in weight. Eight months have passed since the operation. The regulation of the diet and bowels probably had more to do with this result than the operation, as the response was not marked until the intestinal condition was corrected.

Much stress has been laid on nasal disorders as a causative factor in asthma and it is true that this may account for some cases. We should go beyond this and try to determine what produces the nasal condition, unless a sharply defined anatomic malformation exists, causing pressure that only surgical interference can remove. Overlooking this point accounts for many failures to relieve the patient. A cure is effected in some cases in which nasal correction alone is secured, but in others the removal of nasal obstruction does not produce favorable results. So many of these sufferers show such very poor eliminative capacity that little can be expected from the mucous membrane of the nasal passages until the bowels, kidneys and skin are fully performing their functions.

The presence of adenoids in adults has caused asthma in seven patients. That is, seven responded to treatment after removal of adenoids, while many more than seven asthmatics who were the possessors of adenoids showed slight, if any, improvement after operation. The seven that did respond, however, made it worth while. In any event, the adenoid required attention.

- The next class of cases has been little studied. 
CASE 7.-Miss B., aged 28, had a negative family history. Menstruation began at the age of 13 . There was marked dysmenorrhea after the first six months. The attack always preceded the flow by three or four days and abated when the flow was established. A dilatation eight months ago, repeated three months later by the family physician, has resulted in disappearance of the asthma. Menstruation is now practically normal.

Out of four other patients with similar histories only one has responded. Improvement in two out of five, however, made the result worth the effort. The question may be asked: Is the uterus, in patients in whom such treatment is unsuccessful, in reflex spasm from irritation of another center? I am not a gynecologist or a neurologist.

Reports are being made from time to time of successful results following the use of vaccines, and the manufacturers of biologic products are continually suggesting the use of vaccines in this affection, while much disappointment is being experienced as a result. Some cases, no doubt, have cleared up following the use of vaccine; not a vaccine used directly against asthma, but one that was indicated to meet another infection, the asthma, no doubt, being a reflex result.

As headache, backache and numerous other afflictions have proved to be but symptoms, I would suggest, reasoning irom my own experience, that the consideration of asthma as a disease be dropped and the presence of asthma taken to be a symptom reflex. Instead of loading our patients with morphin and everything else in the U. S. P. in an effort to find a cure, we should take a little time between attacks and also during the paroxysm to study and examine these sufferers in a thorough and systematic manner and to obtain a full record of their histories from the date of their birth to the time they put themselves under our care. An inquiry into the menstrual history of female patients and into the condition of the alimentary tract in both sexes should be thorough. The urine examinations should have a complete record as to indican, urea, etc. There will be much disappointment and discouragement, but if the cause can be located once in ten times the result will be worth the time spent; and a record of the case well written ard published will add one more link to the chain that will some day be almost complete as to possible causes, and we can at least tell our patients what produces the asthma, even though we cannot cure it. The treatment very often lies in preventing the attacks, hence is in the hands of the patients themselves and they need rigid instruction.

\section{DIAGNOSIS OF WHOOPING-COUGH BY THF COMPLEMENT-DEVIATION TEST}

PRELIMINARY NOTE *

ALFRED FRIEDLANDER, M.D.
E. A. WAGNER, M.D.
CINCINNATI

The purpose of this preliminary report is to record the fact that we have succeeded in making the diagnosis of whooping-cough in all stages-catarrhal, paroxysmal and convalescent-by means of the complement-deviation test.

We desire also to describe our technic as thus far developed, and to tabulate the results obtained. Several

- From the laboratory of the Cincinnati Hospital, Dr. Paul $G$. Woolley, Director. details of the work are reserved for future communication.

When Bordet and Gengou ${ }^{1}$ described the bacillus of whooping-cough in 1906, they used the complementdeviation test to control their bacteriologic findings. From this time on, it has been known that the blood of patients in the late stages of pertussis, during convalescence and for some time thereafter would give a positive test, showing definite deviation of complement. More recently it has been shown that in the later stages of atypical cases of pertussis, that is, paroxysmal cough without a whoop, it is possible to determine the specific pertussis character of the infection by means of this test. ${ }^{2}$

Several observers have denied the possibility of making a diagnosis of pertussis, even during the height of a typical attack or directly after convalescence by means of the complement-deviation test. Working in the Royal Serological Institute of Vienna, Bächer and Menschikof $^{3}$ report that in twenty-seven cases of pertussis, moderate and severe, in the height of the attack and in convalescence, attempts were made to obtain positive complement-deviation reactions, without success in a single case. Only after vaccines, prepared from pure cultures of the Bordet-Gengou bacillus were given, was the test ever positive.

Commenting on these and other similar findings, Bordet ${ }^{4}$ himself says, "I repeat that the power (of fixing the complement) is not seen early. In general, it does not show itself markedly till toward the period of convalescence or cure."

Netter and Weil $1^{5}$ bave reached practically the same conclusion. They find that the test is constantly positive by the end of the second week of the paroxysmal stage. In the catarrhal stage they were unable to obtain a positive reaction. Of sixteen cases examined during the first week of the whoop, not one gave a positive reaction. They therefore conclude that the test has no diagnostic value in the early stages. This would appear to be the consensus of opinion at this time. It should be noted here that Manicatide ${ }^{\mathfrak{\theta}}$ has recently announced that he has secured positive complement-deviation tests in a series of cases, using as antigen a so-called Zbacillus isolated by him. Confirmation of this assertion is apparently lacking, as are also details as to period of attack in which the positive finds occurred.

Our own results lead us to the opinion that the complement-deviation test is of the very greatest value in the diagnosis of whooping-cough. With the method we have used, it has been possible to make a diagnosis of pertussis in the catarrhal stage (subsequently confirmed by the elinical history). Nine patients were admitted to the hospital just as the children were beginning to whoop; that is, in the first days of the paroxvsmal stage. Our test was positive in all of these cases.

It will be recognized that the early diagnosis of such a scourge as whooping-cough, particularly when occurring in institutions in which children are segregated in large numbers, is a matter of great importance. Again, it is a matter of record that the success of vaccine therapy depends in large measure on the time of its

1. Bordet and Gengou : Ann. de l'Inst. Pasteur, 1906, xx, 731 2. Bordet and Gengou : Centralbl. f. Bakteriol, Abt. 1, Orig., 1911, lviil, 57. Bordet and Rrunard : Bull. Acad. roy. de méd. de (a) ig., 1912, lxi, 218 .

4. Bordet: Centralbl. f. Bakteriol., Abt. 1, Orig., 1912, 1xvi, 276.

5. Netter and Weil : Compt. rend., Soc. de Biol., 1913, lxxir, 236.

6. Manicatide : Ztschr f. Kinderheilk., 1913, vii, 226. 